\title{
Weathering product of granite as a possible source of strategic mineral
}

\begin{abstract}
Several soil samples and granite bedrock samples from Cameron Highlands were analyzed for its elemental concentration using instrumental neutron activation analysis. A total of 34 elements were identified and their concentrations were determined in a single analytical session. Enrichment factor of elemental concentration in the top soil were computed. The findings indicate certain elements were enriched that may facilitates economic beneficiation.
\end{abstract}

Keyword: Elemental concentration; Enrichment factor; INAA; Cameron Highlands Malaysia 